\title{
Current Status and Issues Regarding Transitional Health Care for Adults and Young Adults with Special Health Care Needs in Japan
}

\author{
Hiroyuki Ariyasu and Takashi Akamizu
}

\begin{abstract}
:
With the progress of medical care in recent years, the prognosis of intractable diseases of childhood onset has markedly improved. Young adults with special health care needs require continuous medical support throughout their lifetimes. To provide them with optimal health care services, a smooth transition from the pediatric medical system to the adult one is essential. However, in Japan many adult health providers are not sufficiently prepared to care for these patients, due both to limited opportunities to gain up-to-date medical knowledge on transitional health care and a lack of familiarity with the medical treatment of childhood-onset chronic diseases. In this review, we discuss current issues in transitional health care in Japan from an internist's viewpoint.
\end{abstract}

Key words: transitional health care, adults and young adults with special care needs

(Intern Med 57: 1337-1344, 2018)

(DOI: 10.2169/internalmedicine.9740-17)

\section{Transitional Health Care}

With the progress of medical care in recent years, the prognosis of intractable diseases of childhood onset has improved tremendously. More than $95 \%$ of children with childhood-onset chronic diseases (excluding malignant neoplasms) survive to adulthood in Japan. However, many patients with such diseases are not cured, and they must therefore continue to receive treatment for their conditions and any new complications throughout their lifetimes. In addition, patients often suffer from medical conditions unrelated to their chronic disease, so medical services after adulthood should be delivered by adult health care providers.

"Medical transition" is defined as the process of moving from a pediatric medical system to an adult one. Transfer refers to the actual point in time at which responsibility for patient health care is handed off to the adult health care providers $(1,2)$. However, neither internists nor pediatricians provide adequate care to adults or young adults with special health care needs in Japan. In order to facilitate this transition, it is crucial that health care providers, especially pedia- tricians and internists, accurately share information about patients' medical and social problems and recognize the value of comprehensive medical care.

In this review, we discuss the current status and issues regarding transitional health care in Japan.

\section{Current Status and Issues Regarding Transitional Health Care in Japan}

\section{A) From the standpoint of the patient and their fam- ily, and the pediatrician}

Epidemiological research conducted by the Ministry of Health, Labor, and Welfare of Japan revealed that, in 2012, there were approximately 120,000 Japanese patients with childhood-onset chronic diseases, such as growth hormone deficiency, cretinism, congenital heart disease and lymphocytic leukemia, ranking as follows: endocrine disorders, $32 \%$; chronic heart disease, 16\%; and malignant neoplasms, 14\% (https://www.shouman.jp/research/pdf/15_25/25_02.pdf; site only available in Japanese) (Fig. 1). At present, approximately $20 \%$ of patients with childhood-onset chronic dis-

The First Department of Internal Medicine, Wakayama Medical University, Japan

Received: June 29, 2017; Accepted: September 20, 2017; Advance Publication by J-STAGE: December 27, 2017

Correspondence to Dr. Hiroyuki Ariyasu, ariyasu@wakayama-med.ac.jp 


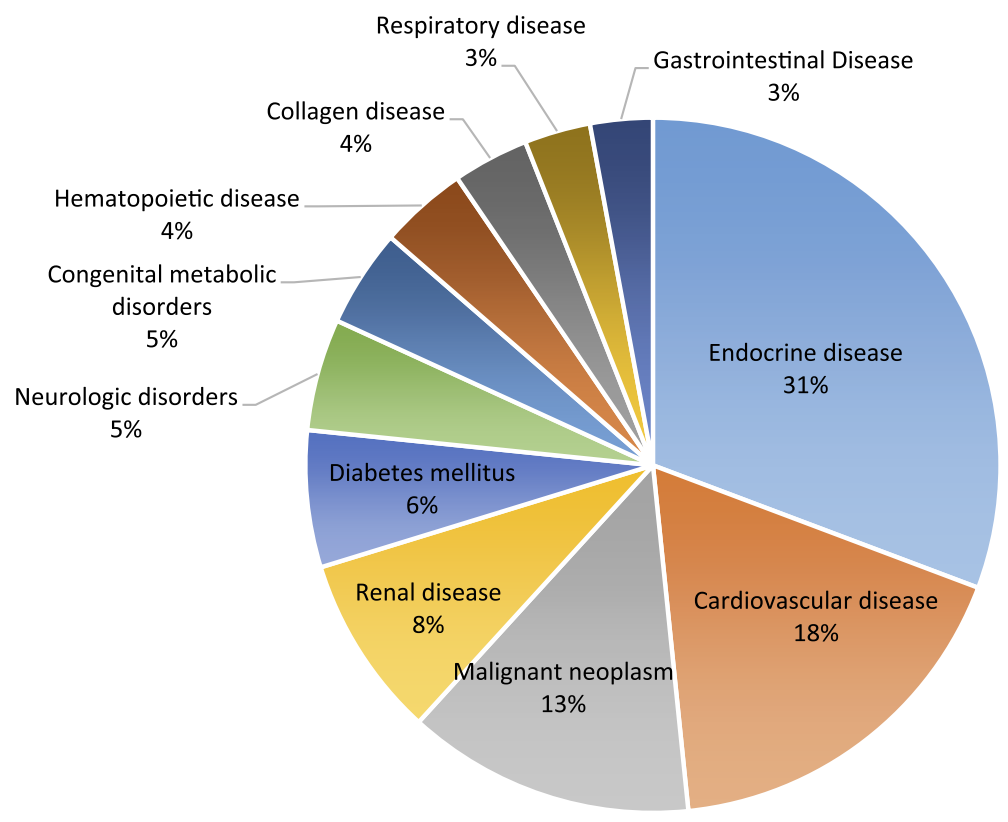

Figure 1. The Childhood-onset Chronic Disease in Japan. From the epidemic survey on childhoodonset intractable disease in Japan in 2012 (arranged), conducted by the Ministry of Health, Labor, and Welfare of Japan.

eases in Japan continue to visit pediatricians even after age 20. In particular, over $40 \%$ of patients with collagen diseases, chronic heart disease, and congenital metabolic abnormalities are still seen in pediatric clinics.

Most patients with childhood-onset intractable disease require long-term outpatient care and/or hospitalization. Their guardians often have a high level of trust in their pediatricians, not only for medical care but also for advice regarding social problems. As a result, establishing new relationships with adult healthcare providers may be psychologically burdensome for patients and their family members, and they may hesitate to transition to the adult health care system. In addition, most pediatricians in Japan are not currently subspecialized and treat patients with all sorts of complex nonsurgical problems. In contrast, most Japanese internists are subspecialists and generally see patients with problems in one specific organ system or limit their practice to one particular area of medical knowledge. Since adults and adolescents with special health care needs sometimes have conditions that affect multiple organs or systems, they are required to visit several centers, such as endocrine, congenital cardiovascular, and cancer centers, and receive separate medical care for each condition. This system may confuse patients and their families, particularly when their preference would be to receive longitudinal care from childhood through adulthood at the same facility. Another factor hampering the transition to adult health care may be the delayed psychological and social development often present in this patient population.

After reaching adulthood, individuals with special health care needs must continue to be treated for their childhoodonset condition while also navigating multiple changes in the social environment, such as employment, marriage, preg- nancy, and childbirth. In addition, they must cope with medical issues unique to adulthood, such as lifestyleoriented diseases (atherosclerosis, heart disease, obesity, type 2 diabetes, etc.) and malignant neoplasms. Internists and obstetricians/gynecologists can appropriately address these conditions, whereas pediatricians cannot. Therefore, in 2014, the Japan Society of Pediatrics published a consensus statement on health care transition for young adults with special health care needs (http://www.jpeds.or.jp/uploads/files/ikouki 2013_12.pdf; site only available in Japanese). The statement stresses that the goal of transitional health care is to provide appropriate and uninterrupted health care services as the individual moves from adolescence to adulthood and to maximize lifelong functioning.

\section{B) From the standpoint of the internist}

In contrast to pediatricians, internists in Japan do not have adequate preparation to accept and manage young adults with special health care needs. Internists are required to accumulate up-to-date medical knowledge and experience regarding childhood-onset chronic diseases that persist into adulthood. Adult health care providers, however, have little experience in treating childhood-onset chronic diseases and have few chances to attend lectures to obtain up-to-date knowledge about transitional medical care; opportunities for training are also quite limited. Therefore, many internists are unfamiliar with the medical treatment of these conditions, and young adults with these conditions have no point of entry into the adult system of care. Furthermore, transitional medicine is a developing science; as such, there is substantial uncertainty about a number of factors, such as how childhood-onset diseases progress with age, when late-onset complications appear, and how medical management should 
Table 1. Chronic Health Conditions in Childhood Cancer Survivor.

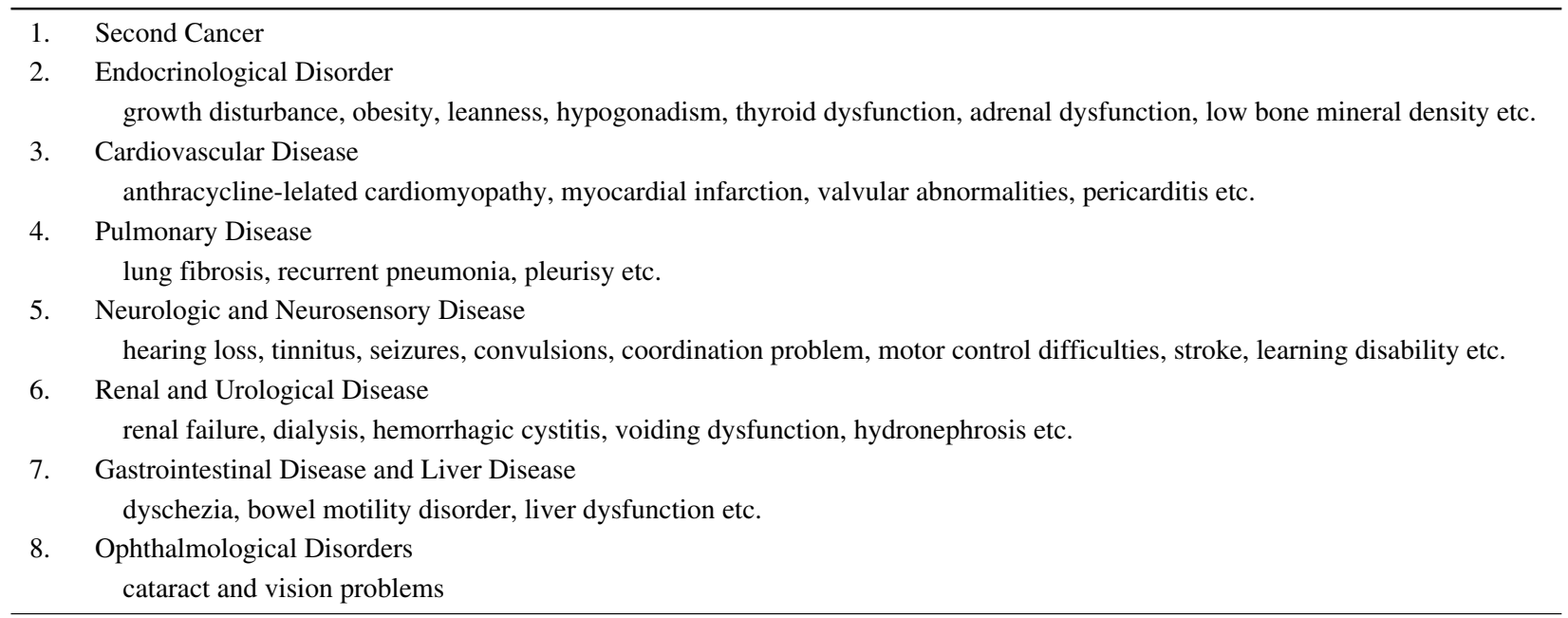

be performed. Although internists understand the importance of their roles in transitional health care, the above factors might prevent the smooth transition of patients into the adult health care system in Japan.

Patients with childhood-onset chronic diseases such as growth hormone deficiency (GHD), thyroid dysfunction, congenital heart disease (CHD), malignant neoplasms, nephrotic syndrome, bronchial asthma, hemophilia, inflammatory bowel disease, and acquired brain conditions, should in principle transfer their care from a pediatrician to an internist when they reach adulthood. Endocrine disorders, heart diseases, and malignant neoplasms are the most common conditions among young adults with special health care needs in Japan. We will now discuss the current status of transitional health care in Japan, with a focus on these three conditions.

\section{Endocrine Disorders and Diabetes Mellitus}

Endocrine disorders are the most prevalent childhoodonset chronic diseases in Japan. Many endocrine disorders require treatment throughout the patient's lifetime, and therefore cooperation between pediatricians and adult endocrinologists is essential for providing optimal health care. Since most patients with endocrine disorders are able to determine their own therapeutic strategy, a smooth and seamless transition can often be achieved relatively easily. However, some patients with endocrine disorders suffer from mental retardation and developmental disorders, and some have multiple diseases. Providing them with optimal care necessitates a medical team with one or more specialists (endocrinologists, psychologists, obstetricians/gynecologists, nephrologists, cardiologists, etc.). In 2012, an epidemiological study of childhood-onset intractable diseases conducted by the Ministry of Health, Labour, and Welfare of Japan revealed that approximately 32,000 patients had an endocrine disorder, in the following rank order: GHD, 41.7\%; thyroid dysfunction, 29.8\%; precocious puberty, 5.0\%; and Turner's syndrome, 4.6\% (https://www.shouman.jp/research/pdf/15_2 5/25_02.pdf; site only available in Japanese). Endocrine health problems are known to often occur in childhood cancer survivors (CCSs) (Table 1) (3). Indeed, Miyoshi reported that $67 \%$ of CCSs in Japan had an endocrinological abnormality (4).

\section{a) $\operatorname{GHD}(5,6)$}

Childhood-onset GHD results has various causes, including genetic abnormalities, injuries at birth, brain tumors, and treatment with irradiation or chemotherapeutic agents. During childhood, GH replacement therapy is performed to improve short stature; in contrast, in adults this therapy addresses an abnormal body composition, impaired physical activity, and a decreased quality of life (7). Since not all patients who are treated with GH replacement therapy during childhood have permanent GHD or require treatment during adulthood, reassessment of the pituitary function is required during the young adult years. When a patient with GHD is transferred from pediatric to adult endocrinologic care, the pediatrician should inform the patient about the name of their disease, precautions in everyday life, and the long-term consequences of GH deficiency in adulthood. The pediatrician should also provide the endocrinologist with accurate medical information, such as the pathogenesis of GHD in each case, the patient's treatment history, and any complications (e.g. gonadotrophic insufficiency, hypothyroidism, adrenal insufficiency, and diabetes insipidus). Since adult GHD is a risk factor for metabolic disorders and cardiovascular disease, it is very important to follow the patient regularly throughout their lifetime.

\section{b) Thyroid disease}

Management of cretinism and childhood-onset Basedow disease requires consistent medical care. Since both diseases are treated similarly in childhood and adulthood, a smooth and seamless transition is relatively easy to achieve as patients mature. 


\section{c) Turner syndrome (8)}

Turner syndrome is a chromosomal condition in which a female is missing an $\mathrm{X}$ chromosome in whole or in part. The most common features of this syndrome are short stature, loss of ovarian function, webbed neck, and cubitus valgus. In Japan, Turner syndrome occurs in approximately 1 in 2,000 to 1 in 2,500 females at birth. Patients with Turner syndrome have numerous medical problems, such as skeletal abnormalities, kidney problems, cardiovascular malformation, diabetes mellitus (DM), hearing disturbance, and reproductive problems. When these issues arise during childhood they should be followed by a medical team consisting of an endocrinologist, cardiologist, nephrologist, audiologist, and fertility specialist throughout the patient's lifetime.

\section{d) Endocrine health problems in CCSs}

CCSs are at risk of late-onset complications due to cancer treatments such as radiation therapy and chemotherapy. Almost $60 \%$ of CCSs have been reported to have at least 1 medical problem (Table 1) $(9,10)$, and $40-67 \%$ were reported to suffer from an endocrine disorder, such as growth retardation, overweight, gonadal dysfunction, bone metabolic disorder, thyroid dysfunction, and adrenal dysfunction $(3,4)$. Since the incidence of late-onset complications increases with age and many appear in adulthood, pediatricians and endocrinologist should educate patients about the frequency with which they should visit a doctor and the reasons for regular follow-up throughout their lifetime. In Japan, the follow-up guidelines for CCSs with endocrine disorders were published by The Japanese Society for Pediatric Endocrinology in 2011, and the guidelines are available for download at http://jspe.umin.jp/medical/files/guide161006.pdf (site only available in Japanese).

\section{e) Diabetes mellitus}

In 2012, an epidemiological study of childhood-onset intractable diseases conducted by the Ministry of Health, Labour and Welfare of Japan revealed that approximately 6,642 patients had DM of the following types: Type $1 \mathrm{DM}$ (T1DM), 82.2\%; Type 2 DM (T2DM), 16.0\%; and DM associated with single-gene defects or other types, $1.8 \%$ (https:// www.shouman.jp/research/pdf/15_25/25_02.pdf; site only available in Japanese). Glycemic control in pediatric patients with T1DM has significantly improved in recent years due to the development of various insulin treatments and improvements in systems for the self-monitoring of blood glucose (11). The Japanese study group of insulin therapy for childhood and adolescent diabetes (JSGIT) reported that the average HbA1c levels in affected patients gradually declined and reached $7.8 \%$ in 2012 .

However, in Japan some young adult patients with T1DM still have poor glycemic control. Glycemic control is known to worsen during adolescence, possibly because the physiological increment in the secretion of $\mathrm{GH}$ and sex steroids leads to insulin resistance. Psychosocial issues during ado- lescence and young adulthood, including independent living, attending college, employment, and alcohol consumption and smoking habits, may also contribute to a loss of control (12). In addition, there may be cases wherein adolescent patients with T1DM have poor diabetes self-management skills because of over-reliance on their parents and inappropriate decision-making.

Poor glycemic control is closely associated with the development of vascular complications, and the National Institute for Health and Care Excellence (NICE) guidelines recommend advising young adult patients with T1DM to maintain HbA1c levels below $7.5 \%$ in order to minimize their long-term risk of developing diabetes-related complications, such as eye, kidney, or nerve damage (the NICE menu of indicators for Quality and Outcome Framework (QOF) is available online at: http://www.nice.org.uk/standards-and-indi cators/qofindicators; Indicator area: Diabetes mellitus, Indicator: NM96). Since the responsibility for daily diabetes management is shifted from the guardians to the patients themselves during adolescence, healthcare providers should help adolescent patients develop diabetes-related knowledge and self-management skills and assist them in utilizing medical care. The goals of a successful transition are to avoid short- and long-term complications of DM, to maintain both mental and physical health, and to live a life that is as similar as possible to that of individuals without diabetes.

\section{Cardiovascular Diseases $(2,13)$}

In Japan, over 10,000 infants with CHD are born each year, and the incidence of tetralogy of Fallot and ventricular septal defect is high (13). Recent epidemiological research has revealed that around $85 \%$ of Japanese patients with CHD reach adulthood, largely due to advances in pediatric cardiac surgery, so the number of adult CHD patients is growing rapidly and now exceeds that of pediatric CHD patients (14). There are estimated to be over 450,000 adult patients with CHD in Japan, and this number is expected to increase by around 10,000 patients annually (14). Many patients with repaired CHD have residual structural and functional cardiovascular problems. In addition, they have emerging complications, such as congestive heart failure, symptomatic arrhythmia, cyanosis, thromboembolism, liver cirrhosis, and protein-losing enteropathy. Since regular, lifelong follow-up is required, adult patients with CHD need to transition from pediatric- to adult-centered care. Although Japanese adult cardiologists understand the need for their involvement in treating adult CHD, most adult patients continue to be followed by pediatric cardiologists (15). Adult cardiologists must accumulate experience with adult CHD and managing symptomatic arrhythmias, pulmonary hypertension, cyanosis, and other conditions. However, many adult cardiologists remain unfamiliar with the medical treatment of adult CHD because the majority of their daily work involves conditions characterized by normal hemodynamics, 
such as ischemic heart disease, congestive heart failure, arrhythmia, etc. In addition, the pathology of adult GHD becomes more complicated if the patient also develops adultonset cardiovascular diseases, such as valve stenosis and/or coronary artery lesions due to hypertension, DM, or dyslipidemia. On reaching adulthood, several additional issues, such as reoperation for CHD, pregnancy, and non-cardiac surgery, arise in patients with adult CHD. Such problems place most patients with repaired $\mathrm{CHD}$ at increased risk of anesthesia-, surgery-, and delivery-related complications. It is therefore important to share accurate medical information, such as the pathogenesis of CHD, treatment history, hemodynamic status, and complications among health care providers.

For a smooth transition from a pediatrician to adult cardiologist, patients' understanding of their heart disease is essential. However, some patients with adult CHD stop their regular medical visits after transitioning from pediatrics to internal medicine, probably because they have insufficient knowledge about the name of their heart disease, the reasons for follow-up, and the symptoms that reflect deterioration of their condition (16).

\section{Malignant Neoplasms: Late Effects Emerging in Adulthood}

An epidemiological study of childhood-onset intractable diseases conducted by the Ministry of Health, Labour and Welfare of Japan revealed that, in 2012, there were approximately 14,000 pediatric patients with cancer. The most common neoplasms, in order, were as follows: hematological neoplasms, 41.2\%; intracranial neoplasms, $12.1 \%$; and neuroblastoma, 5.5\% (https://www.shouman.jp/research/pdf/15_ 25/25_02.pdf; site only available in Japanese). Recent improvements in diagnosing and treating childhood-onset malignant neoplasms have resulted in the long-term survival. It has been reported that the 10 -year relative survival rate for pediatric cancer in Japan is $73.2 \%$ in boys and $79.3 \%$ in girls (http://ganjoho.jp/reg_stat/statistics/stat/summary.html; site only available in Japanese), and about 1 of every 700 adults between the age of 20 and 39 years is a CCS.

However, with improvements in the survival rate, a new problem has arisen. Among CCSs, approximately $60 \%$ have reported health problems caused by chemotherapy or radiation therapy (Table 1) (9). These treatment-related complications or late effects include second cancer, endocrine disorders, renal dysfunction, cardiovascular disease, musculoskeletal problems, and neurologic conditions, with endocrine disorders being the most common $(3,17)$. The incidence of late effects increases with age and does not appear to plateau, with a value of approximately $50 \%$ at 10 years after and $65 \%$ at 25 years after the diagnosis (10). In addition, late effects such as cardiomyopathy due to anthracycline exposure and second cancer may be asymptomatic for a long period of time. General physicians and/or family doctors are expected to play a principal role in the medical sur- veillance of CCSs, and they should obtain accurate medical information, such as the name of the disease, the age of onset, the patient's treatment history (including chemotherapy and radiation therapy protocols), and complications. If cancer treatment is completed during early childhood, some patients may not be informed of their clinical diagnosis and treatment protocol and may not learn about potential late effects; as a result, their physician may underestimate their risk of complications and may not be able to provide optimal medical care. In 2003, the Children's Oncology Group (COG) in the United States published the Long-Term Follow-Up Guidelines for Survivors of Childhood, Adolescent, and Young Adult Cancers (COG-LTFU Guidelines). These guidelines provide recommendations for screening and managing the late effects of pediatric cancer therapy and are available for download at www.survivorshipguidelines.org (18). In Japan, long-term follow-up guidelines for CCSs were published and are available for download at htt p://jplsg.jp/menu11_contents/index_menu_11.htm. Furthermore, the COG pointed out that some CCSs may be at risk of social problems, such as higher health care costs, lower household income, lower educational achievement, and unemployment (19). Since CCSs may require more resources and services than other young people in order to optimize their health, improvement of social security is essential.

\section{Regional Disparities in Transitional Health Care Resources}

There are considerable regional disparities in health care resources across Japan. For example, there are about 2,100 endocrinologists who are board-certified by The Japan Endocrine Society; as a result, there is approximately 1 boardcertified endocrinologist per 60,000 people in Japan, a rate that is comparable to those in other developed nations. However, almost one-third of these endocrinologists work in the greater Tokyo area, and outside the capital, they tend to be concentrated in urban rather than rural areas. Although it would be ideal if patients with intractable diseases could access health care centers locally, currently in Japan, some patients living in rural areas must travel long distances to receive care, an inconvenience that is due to regional disparities in health care resources. Given this situation, local general internists are expected to play a principal role in the routine medical check-ups of patients who cannot frequently visit specialized health care centers in distant areas and who consult with board-certified subspecialists in urban areas as necessary through video-phone calls or internet calls. In addition, developing treatment guidelines and standardizing therapeutic methods may help reduce regional variations in care that affect the management of childhood-onset chronic disease. 


\section{Table 2. Keys for Realizing the Smooth Transition from Pediatrics to Adult Health Care System.}

1. Patients and their family members

a) Development of adequate health literacy

b) Patients' active participation in health care decision-making

2. Health care providers

a) Respect for patients' autonomy

b) Establishment of the guidelines for lifetime management of childhood-onset chronic disease

c) Organization of a medical team consists of a variety of professionals

d) Sharing the accurate medical information about a patient among medical participants

e) Acquisition of current knowledge of contemporary treatment of childhood-onset chronic disease

3. Social security
a) Reduction of the burden of medical costs for essential medical services
- Enrichment of publicly funded health care
- Improvement of private medical insurance
b) Support for education training and employment

\section{Achievement of an Uninterrupted Transition (Table 2)}

For a smooth and seamless transition, respect for patients' autonomy is an ethical principle. Based on that principle, it is essential for patients, their family, and medical participants to cooperate to achieve three main goals. The first is to ensure that patients have a sufficient understanding of their illness. Prior to the transition period, the pediatrician should create a written health care transition plan and provide each patient with special health care needs with detailed information about the name of their disease, treatment history, precautions in everyday life, late-onset complications, and other necessary facts. This process will help the patient understand that, as an adult, they must take responsibility for their own medical care. Young adults with special health care needs should gradually become emotionally independent of their parents and act more on their own initiative regarding their illness rather than following their parents' advice. However, as there are interindividual differences in emotional and physical growth, no single approach will succeed in ensuring a smooth transition and optimal medical service for all patients. Since emotional instability is often seen during the teenage years, it is best to avoid transfer into the adult system of care during this period.

The second goal is to accurately share patients' medical information among medical participants. Since chronic diseases in young adults with special health care needs often involve multiple organ systems, it is important to organize a multidisciplinary medical team as described above. Team members should all be familiar with the patient's medical information, including the pathogenesis of the original disease and any past treatments or complications. Standardization of diagnostic and treatment methods will require the establishment of guidelines and up-to-date educational programs. Epidemiological analyses of the natural history of childhood-onset chronic diseases and the long-term effects of their treatment are also needed.
The third goal is to improve the social security system. Some young adults with special health care needs may suffer from long-term physical and psychological difficulties, which may result in lower educational achievement and unemployment. In addition, they are likely to have higher health care costs, and the financial burden of treatment is one of the main reasons that they stop seeking follow-up. Therefore, improvement of social security is essential to provide optimal health care throughout these patients' lifetimes.

\section{Conclusion}

The aim of transitional health care, which is critical when adolescents with special health care needs become adults, is to provide developmentally optimal health care services while respecting patient autonomy. Compared to pediatricians, internal medicine practitioners in Japan are frequently subspecialized (Fig. 2). The transition to the subspecialized adult health care system seems to be unpleasant for patients and their family members, given their existing familiarity with the pediatric health care system. However, it is a necessary step in order to enable patients to obtain appropriate and uninterrupted lifelong health care services. Thus, medical providers must politely explain the need for this transition to patients and their family members, from the patients' standpoint. A general internist or a board-certificated senior fellow of the Japanese Society of Internal Medicine with experience and knowledge in many medical fields is expected to play a principal role in the routine medical check-up of young adults with special health care needs, and these patients should consult with board-certified subspecialists as necessary. In addition, there are problems to be addressed, including the social security system, regional disparities in health care resource, and shortages of board-certified subspecialists and health care providers. These issues may be mitigated by reducing regional variations in care through defining treatment guidelines and standardizing therapeutic methods. Ultimately, a successful transition to adult-centered care will require close communication among pediatricians, 


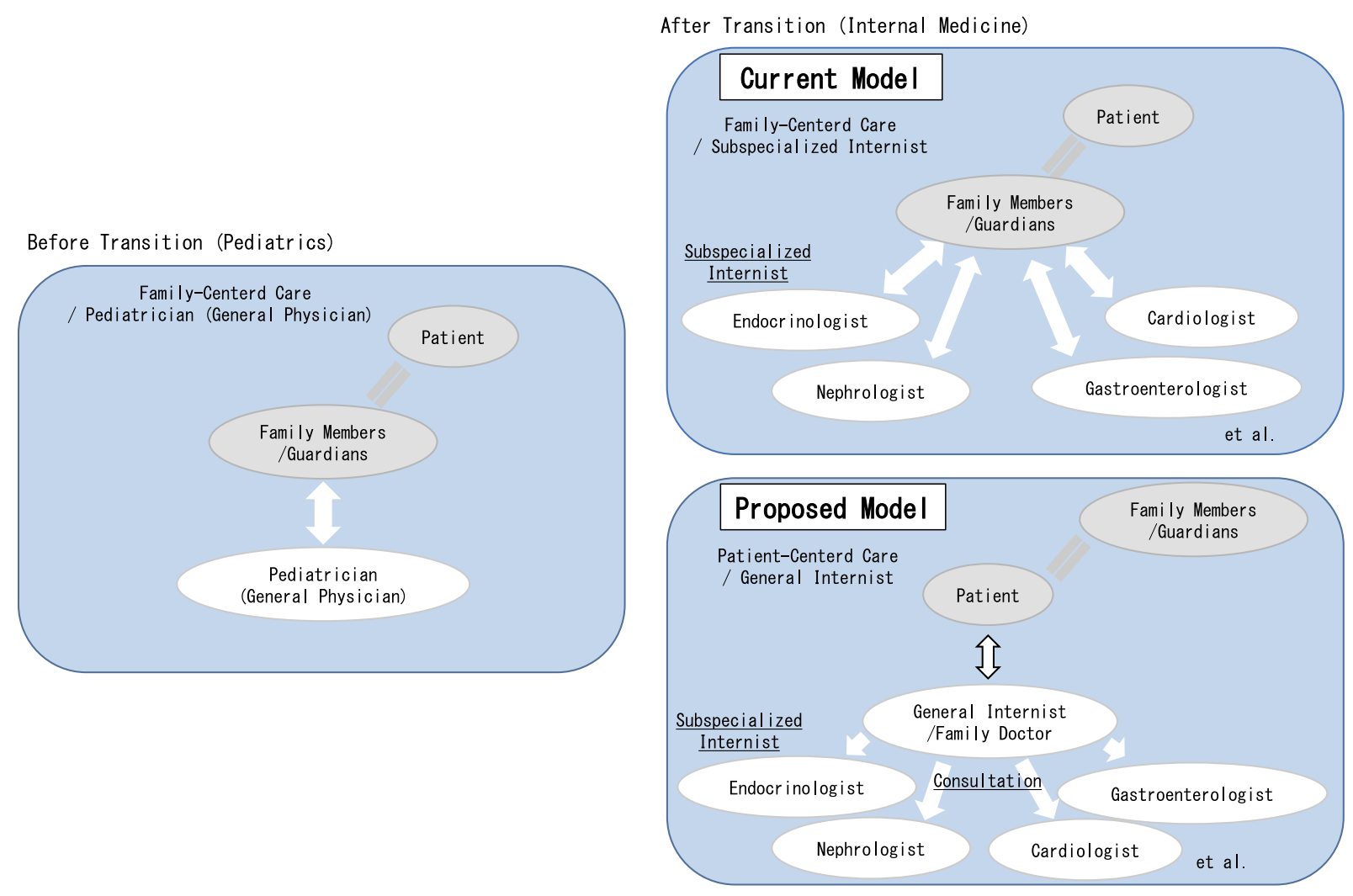

Figure 2. Transitional Care for Adults/Young Adults with Special Healthcare Needs in Japan.

general physicians, subspecialized internists, and patients and their families.

The authors state that they have no Conflict of Interest (COI).

\section{References}

1. American Academy of Pediatrics, American Academy of Family Physicians, American College of Physicians-American Society of Internal Medicine. A consensus statement on health care transitions for young adults with special health care needs. Pediatrics 110: 1304-1306, 2002.

2. Sable C, Foster E, Uzark K, et al. Best practices in managing transition to adulthood for adolescents with congenital heart disease: the transition process and medical and psychosocial issues: a scientific statement from the American Heart Association. Circulation 123: 1454-1485, 2011.

3. Patterson BC, Wasilewski-Masker K, Ryerson AB, Mertens A, Meacham L. Endocrine health problems detected in 519 patients evaluated in a pediatric cancer survivor program. J Clin Endocrinol Metab 97: 810-818, 2012.

4. Miyoshi Y, Ohta H, Hashii Y, et al. Endocrinological analysis of 122 Japanese childhood cancer survivors in a single hospital. Endocr J 55: 1055-1063, 2008.

5. Courtillot C, Baudoin R, Du Souich T, et al. Monocentric study of 112 consecutive patients with childhood onset GH deficiency around and after transition. Eur J Endocrinol 169: 587-596, 2013.

6. Stanhope R. Transition from pediatric to adult endocrinology: hypopituitarism. Growth Horm IGF Res 14(Suppl A): S85-S88, 2004.

7. Giannoulis MG, Martin FC, Nair KS, Umpleby AM, Sonksen P. Hormone replacement therapy and physical function in healthy older men. Time to talk hormones? Endocr Rev 33: 314-377, 2012.
8. Rubin KR. Turner syndrome: transition from pediatrics to adulthood. Endocr Pract 14: 775-781, 2008.

9. Oeffinger KC, Mertens AC, Sklar CA, et al. Chronic health conditions in adult survivors of childhood cancer. N Engl J Med 355: 1572-1582, 2006.

10. Diller L, Chow EJ, Gurney JG, et al. Chronic disease in the Childhood Cancer Survivor Study cohort: a review of published findings. J Clin Oncol 27: 2339-2355, 2009.

11. Swift PG, Skinner TC, de Beaufort CE, et al. Target setting in intensive insulin management is associated with metabolic control: the Hvidoere childhood diabetes study group centre differences study 2005. Pediatr Diabetes 11: 271-278, 2010.

12. Lyons SK, Libman IM, Sperling MA. Clinical review: diabetes in the adolescent: transitional issues. J Clin Endocrinol Metab 98: 4639-4645, 2013.

13. Guidelines for Management of Congenital Heart Disease in Adults (JCS 2011). www.j-circ.or.jp/guideline/pdf/JCS2011_niwa_h.pdf 2015 (In Japanese).

14. Shiina Y, Toyoda T, Kawasoe Y, et al. Prevalence of adult patients with congenital heart disease in Japan. Int J Cardiol 146: 13-16, 2011.

15. Ochiai R, Yao A, Kinugawa $\mathrm{K}$, et al. Status and future needs of regional adult congenital heart disease centers in Japan. Circ J 75: 2220-2227, 2011.

16. Van Deyk K, Pelgrims E, Troost E, et al. Adolescents' understanding of their congenital heart disease on transfer to adult-focused care. Am J Cardiol 106: 1803-1807, 2010.

17. Sklar CA. Overview of the effects of cancer therapies: the nature, scale and breadth of the problem. Acta Paediatr Suppl 88: 1-4, 1999.

18. Children's Oncology Group. Long-term follow-up guidelines for survivors of childhood, adolescent, and young adult cancers. version 4.0. Arcadia, CA, 2014.

19. American Academy of Pediatrics. Long-term follow-up care for pediatric cancer survivors. Pediatrics 123: 906-915, 2009. 
The Internal Medicine is an Open Access article distributed under the Creative Commons Attribution-NonCommercial-NoDerivatives 4.0 International License. To view the details of this license, please visit (https://creativecommons.org/licenses/ by-nc-nd/4.0/).

(C) 2018 The Japanese Society of Internal Medicine

Intern Med 57: 1337-1344, 2018 